\title{
UN COMENTARIO AL CASO KALANKE
}

\section{Una decisión controvertida}

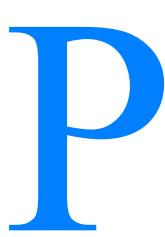

ara quien piense que la bondad de una decisión judicial está en función del mayor o menor grado de aceptación que suscite en la comunidad jurídica y, en general, en la opinión pública, la reciente sentencia del Tribunal de Luxemburgo (en octubre de 1995), a propósito del denominado «caso Kalanke», ha de constituir, sin duda, un ejemplo de decisión equivocada. A decir verdad, el abogado general -Giuseppe Tesauro-, al proponer al tribunal que adoptara la decisión que, en efecto, este último tomó, era plenamente consciente de que «las consideraciones expuestas (...) y la conclusión a que hemos llegado no cuadran con muchas tomas de posición de numerosas instancias» (entre otras, una reciente del parlamento europeo), pero consideraba que era un «deber»-suyo y del tribunal«resistir a la tentación de seguir la corriente dominante» [puntos 26 y 27].

Es, por ello, prácticamente seguro (digamos que en aplicación de la ley de transitividad) que el Tribunal de Justicia de las Comunidades Europeas, sus quince miembros varones, contaron con la posibilidad de que su decisión no iba a ser bien acogida por numerosos círculos de opinión en los diferentes Estados miembros. Pero es bien probable que no previeran del todo la intensidad de esa reacción negativa: por ejemplo, que no esperaran que uno de los efectos de su sentencia fuera que se pusiera en duda la «legitimidad política» del tribunal (como hizo la ministra española Alberdi en declaraciones a la prensa al día siguiente de conocerse la resolución); que el Parlamento europeo celebrara un debate sobre esa sentencia, con el resultado de que la mayoría de los parlamentarios se inclinó «en contra de la decisión del tribunal y en favor de las acciones positivas» [El País, 26-10-1995]; o que la decisión fuese recibida como una agresión incluso por parte del feminismo moderado: así, por ejemplo, 23 senadoras socialistas, en cabezadas por Victoria Camps, firmaron un artículo en el que afirmaban que esa sentencia era uno de los «despropósitos» que «refuerzan la impresión de 
que los derechos de la mujer son papel mojado y estamos aún lejos de la igualdad» [El País, 26-10-1995].

En todo caso, y prescindiendo de las mayores o menores dotes adivinatorias de los miembros del tribunal, quien se propone analizar el caso con las menores dosis de pasión posible tiende a pensar -o, al menos, ése es mi caso- que tanto el abogado general como los jueces europeos debieron tener muy buenas razones para actuar como lo hicieron. Para comprobar hasta qué punto resulta o no confirmada esa presunción, conviene empezar por un breve resumen del caso en cuestión.

\section{El caso Kalanke}

En julio de 1990, la ciudad de Bremen convoca una plaza de jefe de departamento del servicio de espacios verdes. Concurren, entre otros, el señor Kalanke y la señora Glissmann, quienes trabajaban ya en ese departamento (el uno desde 1973 y la otra desde 1975), ambos en calidad de técnicos clasificados con el grado III, de acuerdo con el correspondiente convenio colectivo. Aunque la dirección del servicio propone al señor Kalanke, quien termina por obtener el puesto, después de diversas vicisitudes (el comité de personal se opone a la propuesta en favor de Kalanke y hay un intento de arbitraje que termina con una recomendación en favor de éste último; esa recomendación no es aceptada por el comité de personal, el cual eleva consulta a la comisión de conciliación que se pronuncia en favor de la candidata femenina), es la señora Glismann, en base a una ley del Estado de Bremen que establece que cuando existen dos candidatos con «igual calificación» para acceder a un puesto vacante y las mujeres están subrepresentadas en ese sector (no representan al menos la mitad de los efectivos), debe darse prioridad a la mujer.

El señor Kalanke recurre esta decisión ante los tribunales alemanes, alegando que no se ha tenido en cuenta la superioridad de su calificación profesional y que la ley de Bremen va en contra de normas de superior jerarquía (entre otras, la propia Constitución alemana que, naturalmente, recoge el principio de igualdad de trato). El recurso es rechazado y el caso llega hasta el Tribunal de Trabajo Federal que estima que el régimen de cuotas previsto en aquella ley es conforme con las normas nacionales alemanas -cuya violación alegaba el recurrente-, pero plantea una cuestión prejudicial ante el Tribunal de Justicia de las Comunidades Europeas, para que éste decida sobre si la regulación en cuestión contradice o no el art. 2, apartado 4, de una determinada directiva comunitaria de 1976 (76/207 CEE del Consejo, de 9 de febrero). Ese artículo 2 comienza, en su primer apartado, por establecer que «el principio de igualdad de trato (...) implica la ausencia de toda discriminación basada en el sexo, ya sea directamente, ya indirectamente por referencia, especialmente, al estado matrimonial o familiar»; pero, en su apartado 4, permite a los Estados miembros adoptar y/o 
mantener en vigor las «medidas dirigidas a promover la igualdad de oportunidades entre hombres y mujeres, en particular, remediando las desigualdades de hecho que afecten a las oportunidades de las mujeres en los aspectos contemplados en el articulo primero [acceso al empleo, incluida la promoción, formación profesional, condiciones de trabajo, etc.]».

El abogado general, en su pormenorizado escrito de conclusiones, propone -como antes recordaba- al tribunal que decida la cuestión que se le plantea en el sentido de considerar que la reglamentación nacional a examen es, en efecto, contraria a la directiva de la Comunidad. El tribunal lo hace así, y en su fallo establece que la Directiva «se opone a una reglamentación nacional que, como en este caso, acuerda automáticamente, a iguales calificaciones entre candidatos de diferente sexo considerados en vista de una promoción, una prioridad a los candidatos femeninos en los sectores en que las mujeres están subrepresentadas».

\section{La posición del abogado general y su fundamentación}

La argumentación del abogado general para justificar la conclusión que se acaba de señalar es notablemente compleja, extensa (baste con decir que transcurre a lo largo nada menos que de 28 puntos no siempre breves) y quizás también un poco oscura y elusiva en algún extremo. No resulta por ello nada fácil de sintetizar, pero me parece que lo esencial de la misma se contiene en los siguientes pasos:

(1) El punto de partida, la cuestión a discutir -como se ha dicho-, estriba en si el referido artículo de la ley de Bremen es o no contrario al art. 2, apart. 4, de la directiva comunitaria [punto 12]

(2) Para resolver esa cuestión, surgen tres líneas de argumentación relativamente independientes.

(2.A)

(2.A.1) Contestar en uno u otro sentido a esa pregunta depende de cómo se interprete la expresión «igualdad de oportunidades», utilizada por la directiva como criterio para determinar el ámbito de las medidas positivas en favor de la mujer que los Estados miembros podrían lícitamente adoptar.

(2.A.2) En principio, esa noción podría entenderse bien en el sentido de «igualdad en los puntos de partida» o bien como «igualdad en los puntos de llegada» (o igualdad en cuanto al resultado) [punto 13]

(2.A.3) Pero sólo la primera interpretación resulta aceptable (es decir, «igualdad de oportunidades» ha de entenderse exclusivamente como «igualdad en los puntos de partida»), por las siguientes razones:

(2.A.3.1) El art. 2, aptdo. 4, es una excepción al principio de igualdad de trato (establecida en el aptdo. 1 del mismo artículo) y, puesto que se trata de «una derogación de un derecho individual», ha de interpretarse restrictivamente [punto 23]. 
(2.A.3.2) Esta interpretación restrictiva es la que se recoge también en una anterior decisión del tribunal (de octubre de 1988) en la que se señala que ese artículo de la directiva sólo autoriza tratos que sean «aparentemente discriminatorios» y estén «destinados concretamente a eliminar los obstáculos de hecho que impiden la realización de la igualdad de oportunidades para las mujeres» [punto 15].

(2.A.3.3) El art. 2, aptdo. 4, de la directiva establece un trato preferencial en favor de las mujeres que resulta incompatible con un sistema de cuotas. La razón para ello es como sigue:

(2.A.3.3.1) Ese trato preferencial tiene su razón de ser en una «situación general de desventaja» debida bien a dificultades de hecho provocadas por el doble papel (familiar y profesional) que la mujer desarrolla, o bien a discriminaciones sufridas en el pasado [punto 18].

(2.A.3.3.2) Ahora bien, en el primer caso, tales dificultades no pueden, de hecho, resolverse mediante un sistema de cuotas, sino mediante medidas relativas a la organización del trabajo (por ejemplo, flexibilidad en los horarios de trabajo, estructuras destinadas a la infancia, etc.) [punto 18].

(2.A.3.3.3) Respecto a las discriminaciones del pasado, es cierto que sus «efectos se hacen sentir aún», pero «la acción positiva no puede ser considerada, y aún menos utilizada, como un medio para restaurar, mediante medidas discriminatorias, una situación de igualdad que se ha deteriorado en el pasado». [punto 19].

(2.A.3.4) Esa interpretación no choca tampoco con la recomendación 84/635/ CEE del Consejo, de 13 de diciembre de 1984, relativa a la promoción de acciones positivas en favor de las mujeres, destinadas «a eliminar las desigualdades de hecho de que son objeto las mujeres en la vida profesional, así como a promover la mixtura de sexos en el empleo». La razón en este caso es la siguiente:

(2.A.3.4.1) La recomendación no contiene una «definición de la acción positiva, sino solamente una lista de objetivos a los que tal acción puede tender». «La acción en favor del desarrollo, de la presencia y participación de las mujeres en todos los sectores, en todas las profesiones y en todos los niveles de responsabilidad está, ella misma, formulada en términos de incentivación, pero no ciertamente de preferencia mecánica» [punto 20].

(2.A.4) La reglamentación del Estado de Bremen no está en absoluto destinada a garantizar la igualdad en cuanto a los puntos de partida, sino en cuanto a los resultados, puesto que:

(2.A.4. 1) La circunstancia de que «dos candidatos de sexos diferentes posean calificaciones equivalentes implica, en efecto, y por definición, que ambos poseen, y poseían, iguales oportunidades: se encuentran, pues, en condiciones iguales en la línea de salida» [punto 13]

(2.B)

(2.B.1) La ley de Bremen prevé una medida que no puede justificarse de acuerdo con los principios del Derecho comunitario, ya que: 
(2.B.1.1) Una medida discriminatoria (que atenta contra el principio de igualdad formal de trato) sólo está justificada si respeta el principio de proporcionalidad, esto es, si es necesaria y apropiada para realizar el objetivo perseguido: remover los obstáculos de hecho que se oponen a la realización de la igualdad de oportunidades entre hombres y mujeres [puntos 23 y 20].

(2.B.1.2) Pero la medida en cuestión se dirige sólo a eliminar la subrepresentación de la mujer, no los obstáculos -las causas- que provocan esa subrepresentación [punto 24].

(2.B.1.3) Además, la medida resulta también desproporcionada porque «la subrepresentación de las mujeres en un segmento dado del mercado de trabajo, incluso si constituye una señal de una disparidad, no es necesariamente imputable a una voluntad consumada de marginar a las mujeres. De ahí surge el elemento de arbitrariedad inherente a todo trato preferente reservado, de manera mecánica, al grupo subrepresentado y fundado únicamente sobre este motivo» [punto 24].

(2.C) La medida en cuestión no promueve la verdadera igualdad de la mujer, sino una simple «igualdad numérica» que no es otra cosa que «formal». «Lo que es necesario, en definitiva, es sobre todo un cambio de fondo del modelo económico, social y cultural que está en el origen de la disparidad, cambio que no se realiza ciertamente con cifras o con luchas intelectuales ya desfasadas» [puntos 27 y 28].

(3) Por lo tanto, la reglamentación del Estado de Bremen se opone a la directiva comunitaria.

\section{Crítica de la fundamentación}

Examinemos ahora brevemente los anteriores argumentos, teniendo en cuenta que (como se muestra en el esquema) hay una cierta intersección entre la línea argumentativa (2.A) y (2.B); o, más exactamente, que algunos de los argumentos de (2.B) suministran apoyos para algunos de los argumentos de (2.A).

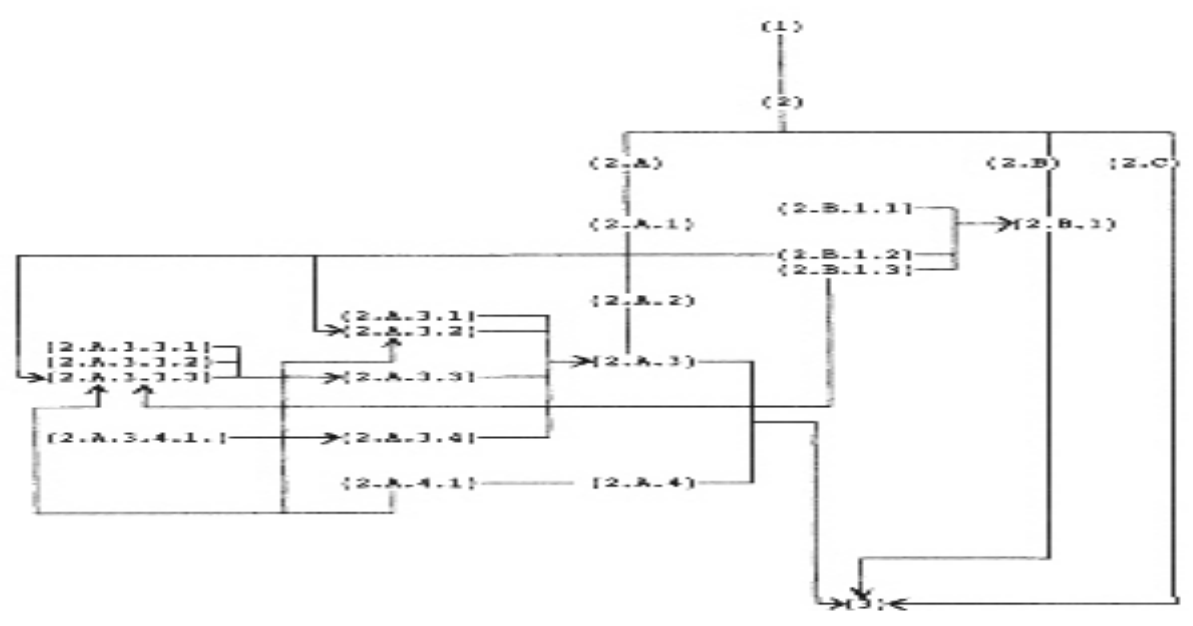


Empiezo por la argumentación contenida en (2.A). En favor de la premisa (2.A.3) el abogado general da, como hemos visto, cuatro argumentos. Los dos primeros contienen razones de innegable peso, pero no parece que ellos solos, sin más argumentos adicionales, permitan justificar -y quizás tampoco lo pretenda el abogado general- la interpretación propuesta. En relación con (2.A.3.1), no basta con sostener que la interpretación de la Directiva debe hacerse de manera restrictiva; se necesitaría también, o sobre todo, precisar "cómo de restrictiva ha de ser esa interpretación». Y con respecto a (2.A.3.2), aun aceptando que el Tribunal debiera seguir la interpretación establecida para el artículo en un caso anterior, con el que el caso Kalanke guarda sólo una analogía de tipo formal ${ }^{1}$ (y es obvio que no necesariamente tiene que ser así: los tribunales, bajo ciertas condiciones, pueden y deben modificar su jurisprudencia), el criterio interpretativo dista de ser claro: no es fácil saber qué significa que una medida es sólo «aparentemente» discriminatoria; uno tendería a pensar que «aparentemente» no puede significar más que «justificadamente», pues me parece innegable -y también se lo ha parecido al abogado general ${ }^{2}$ - que cualquier medida que tenga como base el art. 2, aptdo. 4, de la Directiva implica de hecho una discriminación (esto es, un trato desigual). Ni tampoco si una medida como la prevista en la ley de Bremen está «destinada» a «eliminar los obstáculos de hecho que impiden la realización de la igualdad de oportunidades para las mujeres». Es cierto que el abogado general da razones -los argumentos (2.B.1.2) y (2.A.4.1)- para contestar a esta última pregunta en forma negativa, pero -como luego mostraré- tales razones son, cuando menos, discutibles.

Con respecto a (2.A.3.3), el punto problemático de la argumentación se contiene, naturalmente, en (2.A.3.3.3), esto es, en la cuestión referida a la situación de desventaja en que de hecho se encuentran las mujeres (lo que reconoce el abogado general) debido a las discriminaciones sufridas en el pasado. Y aquí es donde la argumentación del abogado general me parece un tanto confusa y elusiva. La premisa implícita de su razonamiento parece ser ésta: una política de cuotas -o, más en general, una acción positiva- en favor de las mujeres sólo puede aceptarse si: a) resulta un medio eficaz para remover los obstáculos que impiden la existencia de una verdadera igualdad de oportunidades entre hombres y mujeres; y b) es jurídicamente aceptable, en el sentido de que no va contra ningún principio o valor fundamental. Ahora bien, de nuevo, en favor de la no eficacia de esa medida -en concreto, de la prevista por la ley de Bremen- sólo se aportan los argumentos (2.A.4.1) y (2.B.1.2), de los que paso a ocuparme en seguida. Y sobre su carácter

\footnotetext{
${ }^{1}$ Es decir, una analogía que no se refiere al contenido de los casos. Sobre la distinción entre analogía formal y material, véase Manuel Atienza, Sobre la analogía en el Derecho, Madrid, Civitas, 1986.

2 En el punto 11, se puede leer: «La verdad es que toda acción específica en favor de una categoría minoritaria o, en todo caso, vulnerable, se opone al principio de igualdad en sentido formal».
} 
injustificado, el único apoyo que encuentro en todo su escrito ${ }^{3}$ es el argumento (2.B.1.3) que, tal y como lo usa el abogado general, resulta notablemente débil.

(2.A.3.4) es, por razones obvias, un argumento que en ningún caso puede significar un apoyo decisivo en favor de (2.A.3), esto es, de la interpretación de «igualdad de oportunidades» exclusivamente como «igualdad en el punto de partida»: en primer lugar, porque se trata simplemente de una «recomendación»; $y$, en segundo lugar, porque aunque sí lo que en la misma se propusiera fueran únicamente medidas de «incentivación», de ahí no puede seguirse, sin más, la prohibición de cualquier otro tipo de medidas.

Finalmente, en relación con (2.A.4.1) (la razón esgrimida para sostener que la reglamentación del Estado de Bremen no está en absoluto destinada a garantizar la igualdad en cuanto a los puntos de partida, sino en cuanto a los resultados), me parece bastante claro que no constituye propiamente un argumento, sino más bien una falacia (es decir algo que puede parecer un argumento -un buen argumento- sin serlo) basada en una ambigüedad.

Pues el poseer calificaciones equivalentes puede entenderse, en efecto, que significa tener «igualdad de oportunidades» para aspirar al puesto, pero no necesariamente que ambos hayan tenido «igualdad de oportunidades» para llegar hasta ahí, esto es, para alcanzar esa determinada calificación: elque ambos candidatos poseyeran calificaciones equivalentes no supone, por tanto, absolutamente nada en relación a si tuvieron o no igualdad de oportunidades, pues parece obvio que es la segunda noción de «igualdad de oportunidades», y no la primera, la que aquí interesa.

Por lo que se refiere a la segunda línea de argumentación, (2.B), los puntos a discutir son, sin duda, (2.B.1.2) y (2.B.1.3). El primero de estos argumentos -que, como ya se ha visto constituye una pieza fundamental de toda la argumentación- tiene un carácter empírico, pues lo que viene a decir es que una medida como la prevista en la ley de Bremen se dirige (es apta) sólo para eliminar la subrepresentación de la mujer, pero no los obstáculos, las causas, que provocan esa subrepresentación. El abogado general da por descontado que esto es así, pero me parece que ésa es una suposición discutible. Precisamente, el Tribunal de Luxemburgo recoge en su sentencia (aunque sin pronunciarse sobre el mismo) un argumento esgrimido por la jurisdicción de reenvío (el Tribunal de Trabajo Federal) en favor de un régimen de cuota como el que se discute, que «puede contribuir a superar en el futuro las desventajas sufridas actualmente por las mujeres y que perpetúan las desigualdades del pasado, en la medida en que crea el hábito de ver a las mujeres asumir igualmente ciertas funciones más prestigiosas» [punto 14]. Personalmente, no dispongo de ninguna información que permita confirmar o desmentir una tal hipótesis (ni el abogado general ni el tribunal suministran

${ }^{3}$ En el punto 9 hay simplemente una referencia a la discusión contemporánea sobre el particular, pero lo único que se desprende de ahí es que ése es un problema controvertido. 
tampoco ningún dato al respecto), pero diría que, cuando menos, es una afirmación plausible.

(2.B.1.3) es un argumento que, en el discurso del abogado general, aparece en cierto modo en passant, de manera que queda un poco desdibujado e impreciso en cuanto a qué es lo que pretende justificar, esto es, al lugar que ocupa en la argumentación. Tal y como aparece formulado, lo que viene a decir es que la medida prevista por la ley de Bremen resulta arbitraria por que trataría de «compensar» a las mujeres por sufrir una marginación que puede no haber sido provocada de manera voluntaria (se entiende: por los varones). Así expresado, el argumento no tiene, en mi opinión, mucho peso, pues me parece claro que se puede ser responsable -jurídica y moralmente- por las consecuencias no deseadas o no previstas de nuestras acciones.

Finalmente, en relación con la línea argumentativa (2.C), un lector del libro de Bentham Falacias políticas se sentiría, creo yo, predispuesto a considerar que lo que allí se contiene es el tipo de falacia a la que el filósofo utilitarista llamó ad verecundiam y para la que daba esta regla de uso: «cuando se propone una medida a la que, por la razón que fuere, os conviene o complace oponeros (...) mostrad vuestro apoyo a cualquier otra medida que, por la razón que fuere, pueda parecer a vuestros oyentes de superior importancia (...) Por este medio creáis una diversión y apartáis de la odiada medida el favor y atención de la audiencia $\iota^{4}$

\section{5. ¿Una mejor argumentación?}

El apartado anterior arroja, cabría decir, un resultado poco claro, en relación con la hipótesis que planteaba al final del apartado 1. Por un lado, podría decirse que la argumentación del abogado general presenta una serie de puntos débiles más o menos notorios, pero, por otro lado, esas debilidades o no son tan importantes como para quitar fuerza al conjunto de la argumentación o bien podrían subsanarse reformulando los argumentos. Así, por ejemplo, la línea argumentativa (2.C) no pasa de ser una especie de «desahogo» ideológico cuyo papel en la argumentación propiamente dicha no tiene mayor relevancia. En favor de (2.A.4) se podrían dar seguramente argumentos de mucho más peso que (2.A.4.1); en realidad, ésa es una premisa casi indiscutible (pues parece bastante obvio que una ley como la de Bremen se dirige de manera inmediata a alcanzar una igualdad en cuanto a los resultados, aunque mediatamente pretenda también la igualdad en cuanto al punto de partida), de manera que ni siquiera hubiese necesitado de ningún argumento. (2.B.1.2) podría reformularse en el siguiente sentido: quizás no esté claro que las medidas previstas en la ley carezcan de eficacia para remover

4 Jeremy Bentham, Falacias políticas (estudio preliminar de B. Pendás, traducción de J. Ballarín), Centro de Estudios Constitucionales, Madrid, 1990, p. 120. 
los obstáculos que provocan la subrepresentación de las mujeres, pero ciertamente tampoco parece que pueda darse con facilidad una prueba clara de su eficacia; además, para justificar esa medida no bastaría con probar que es eficaz, sino que se necesitaría algo más, a saber, que está justificada, esto es, que no choca con principios fundamentales del Derecho comunitario. El centro de la argumentación se desplaza así hacia una cuestión que en el discurso del abogado general está, a pesar de las apariencias (ya se ha dicho que en los primeros puntos de su escrito hay una referencia relativamente amplia a la discusión contemporánea sobre la justificación o no de la discriminación inversa), básicamente eludida. El único argumento que hace al caso es (2.B.1.3) que, sin embargo, tal y como aparece formulado, no sirve de mucho. ¿Pero podría reformularse de manera que suministrara un apoyo decisivo a la tesis del abogado general? Probablemente sí.

Lo que vendría a decir ahora el argumento -y quizás fuera eso lo que pretendió decir el abogado general- es que la ley de Bremen -y, en general, cualquier sistema de discriminación inversa- establece un supuesto de responsabilidad indirecta y objetiva carente de justificación. Pues, por ejemplo en este caso, el señor Kalanke sufre un perjuicio en razón de la discriminación de la que fueron objeto en el pasado las mujeres; esto es, por acciones que él no realizó ni, por tanto, pudo evitar de ninguna forma: en definitiva, se le trata peor que a otro individuo no en razón de alguna circunstancia que esté bajo su control, sino por su pertenencia -obviamente no voluntaria- al grupo de los varones. Este es, sin duda, el argumento de más peso que cabe esgrimir contra cualquier medida que suponga una discriminación inversa (y que, formulado quizás de otra manera, lo que viene a decir es que los titulares de los derechos son exclusivamente los individuos y no los grupos ${ }^{5}$ ).

Sin embargo, a mi juicio (y me parece que en esto discrepo de la tesis de fondo del abogado general), no tiene por qué considerarse como una razón para excluir absolutamente una política de cuotas -o, en general, medidas de discriminación inversa-, aunque sí tiene fuerza suficiente como para exigir que su utilización transcurra dentro de límites que la ley de Bremen quizás haya transgredido.

En mi opinión, los dos principios que regulan (en el sentido de que permiten justificar) una medida de discriminación inversa podrían formularse así:

1) La utilización de una medida de discriminación inversa solo está justificada si:

${ }^{5}$ Una excelente, y breve, exposición y evaluación de los diferentes argumentos que suelen darse en contra de las políticas de discriminación inversa puede encontrarse en el artículo de Alfonso Ruiz Miguel, «Discriminación inversa e igualdad», en Amelia Valcárcel (comp.), El concepto de igualdad, Editorial Pablo Iglesias, Madrid, 1994, pp. 77-93. 
a) Resulta razonablemente eficaz y eficiente para alcanzar el objetivo perseguido: puede presumirse que se logrará el objetivo y sin un coste excesivo;

b) Se trata de un objetivo socialmente importante. En el caso de una medida de discriminación inversa que beneficia a las mujeres, tendría que darse el caso de que esa medida afectara positivamente, al menos en forma indirecta, a un grupo extenso de mujeres y que no redundara básicamente en beneficio de las mejor situadas socialmente.

c) No existe ninguna otra medida alternativa para alcanzar ese objetivo y que afecte en menor grado a derechos fundamentales de los individuos.

2) Quien pretende justificar (utilizar) una medida de discriminación inversa corre con la carga de la argumentación; es decir, es él quien debe probar que se dan las anteriores circunstancias a), b) y c).

Si ahora aplicáramos el anterior test a la medida propuesta por la ley de Bremen, mi opinión es que resulta bastante dudoso que logre superar ambos principios, a diferencia, por cierto, de lo que ocurriría en el caso de la utilización de cuotas para cargos políticos o para ser miembro de un tribunal como el de Luxemburgo. La diferencia fundamental consiste en que en estos últimos dos supuestos, no parece que una política de cuotas lleve consigo el sacrificio del derecho de un individuo: no tiene sentido decir que alguien tiene derecho a ostentar un cargo público o a ser nombrado juez-al menos, en un caso como el del Tribunal de Luxemburgo-, pero sí que lo tiene cuando de lo que se trata es de obtener un determinado puesto de trabajo o de lograr un ascenso.

\section{La sentencia del tribunal de Luxemburgo y su interpretación}

Ahora bien, lo que se contiene en la sentencia del Tribunal de Luxemburgo no es una reformulación de la argumentación del abogado general semejante a la que se acaba de efectuar, sino una asunción de la misma, pero presentada en forma considerablemente más sintética. Así, para llegar a la conclusión que ya nos es conocida, la argumentación del Tribunal transcurre por medio de estos tres pasos:

(1) El art. 2, aptdo. 4, de la Directiva ha de interpretarse de manera estricta, pues se trata de la derogación de un derecho individual consagrado por la Directiva [punto 21]. Además, y de acuerdo con la sentencia ya mencionada de 25 de octubre de 1988, ese aptdo. tiene «como fin preciso y limitado autorizar medidas que, aun siendo discriminatorias según las apariencias, se dirigen efectivamente a eliminar o a reducir las desigualdades de hecho que puedan existir en la realidad de la vida social» [punto 18].

(2) Más en concreto, el apartado en cuestión autorizaría medidas nacionales que tienen como objetivo mejorar la capacidad de las mujeres para 
concurrir en el mercado de trabajo y para realizar una carrera en plan de igualdad con los hombres [punto 19]. Pero una reglamentación nacional que «garantiza una prioridad absoluta e incondicionada a la mujer en caso de nombramiento o promoción, va más allá de una promoción de la igualdad de oportunidades y sobrepasa los límites de la excepción prevista en el art. 2, aptdo. 4» [punto 22].

(3) La reglamentación de la ley de Bremen está en este segundo caso, pues «sustituye la promoción de la igualdad de oportunidades contemplada en el art. 2, aptdo. 4, por el resultado al que sólo podría llegarse mediante la puesta en marcha de una tal igualdad de oportunidades» [punto 23].

El problema esencial que plantea la anterior fundamentación -como lo han señalado muchos comentaristas- es que su parquedad hace que no sea fácil determinar con precisión qué debe considerarse como la ratio decidendi del fallo. $\mathrm{O}$, dicho de otra manera, lo que el Tribunal considera prohibido es un sistema que, dadas determinadas condiciones, asigna automáticamente una prioridad a las mujeres. Pero esto parece querer decir que bajo las mismas condiciones (promoción a un puesto de trabajo, subrepresentación femenina e igual calificación de los candidatos) podría ser aceptable un sistema que conceda prioridad a las mujeres, pero siempre que no sea de manera automática. Y para entender lo que este último criterio significa, conviene aclarar qué deba entenderse por «igual calificación» y por asignación «automática», puesto que los otros términos, «promoción a un puesto de trabajo» $\mathrm{y}$ «subrepresentación femenina» no ofrecen dudas.

«Igual calificación» es, obviamente, un concepto comparativo y, por tanto, relativo. Por ejemplo, A y B pueden tener la misma calificación con respecto a X y a Y, pero distinta con respecto a Z. Para aclarar el concepto se necesita, por tanto, precisar cuáles son las propiedades que se usan en la comparación y, también, de qué tipo de propiedades se trata. En el caso que nos ocupa, la comparación se establece únicamente en relación con una propiedad (haber sido o no clasificado en el nivel III de un determinado convenio colectivo) que además es considerablemente genérica (se está o no en el nivel, pero dentro del mismo no se establece ninguna gradación). Por supuesto, las autoridades administrativas o jurisdiccionales podrían haber hecho otra interpretación de la expresión «igual calificación» que aparece en la ley de Bremen. Podrían haber dicho que para determinar que dos candidatos tienen una «igual calificación» no basta con considerar esa propiedad, sino que debían tenerse en cuenta otras como la mayor o menor experiencia, los informes de sus superiores o de especialistas en la materia, etc. De haber sido así, es posible que el tribunal hubiese dicho que la ley en cuestión no era contraria a la Directiva, pero el caso es que no fue eso lo que ocurrió, y el Tribunal de Luxemburgo no tenía competencia para imponer a un Estado miembro una determinada interpretación de sus normas. Por lo tanto, el tribunal no tenía, por ese lado, ninguna capacidad de maniobra. 
Ahora bien, el mismo efecto que podría haber producido esa otra interpretación del término «igual calificación» puede lograrse si a las tres condiciones que contiene la ley alemana se le añade la de que la prioridad en favor de la mujer no sea «automática» o «absoluta e incondicionada» como también se dice en otro de los puntos de la sentencia. O sea, lo que el tribunal parece querer decir es que la asignación de una ventaja a las mujeres para lograr una determinada promoción puede ser aceptable si no se considera únicamente la igualdad de oportunidades entendida en el primero de los sentidos (esto es, de manera completamente genérica), sino también otra serie de elementos, de propiedades, que el tribunal no precisa, pero que deberían ser de tal naturaleza que permitieran efectuar algo así como una «ponderación», un «equilibrio», etc. (pues parece que éstas son, justamente, las ideas que se oponen a «automática»). Dicho de otra manera, a lo que apunta el tribunal es a que la Directiva podría ser compatible con un sistema de objetivos o goals que asigne una cierta puntuación al hecho de ser mujer, como parte de un determinado baremo que incluya un número suficientemente elevado -y quizás graduable- de items, de manera tal que el hecho de ser varón no se convierta en una característica prácticamente insuperable para lograr la promoción.

Interpretada de esta última manera la ratio decidendi del fallo, la sentencia del Tribunal de Luxemburgo es, en mi opinión, una decisión jurídica y moralmente correcta que, además, no resulta incompatible con una política de discriminación inversa (o de acción afirmativa) en favor de la mujer, y ni siquiera con todo sistema de cuotas. 